\title{
Hacia nuevos modelos de enseñanza-aprendizaje en ciencias de la salud
}

Jornada de la Sociedad de Educación Médica de Euskadi (SEMDE)

Bilbao, 22 de noviembre de 2010

\section{Ponencia}

\section{Simulación como solución a las nuevas necesi- dades en el mundo sanitario}

\section{J.A. Muro Sans}

Diplomado en Enfermería (EUI Santa Madrona). Licenciado en Enfermería (London City University). Education manager de Medical Simulator. International Adjunct Faculty for METI. Londres.

Existe una creciente preocupación por la seguridad del paciente en el ámbito hospitalario y extrahospitalario, se han empezado a plantear cuestiones sobre la eficacia de la formación de los profesionales de la salud. Esta preocupación cuestiona la eficiencia de la educación durante el curso universitario y plantea si se ofrece suficiente experiencia práctica a los estudiantes universitarios antes de que se enfrenten a la profesión sanitaria.

Los conocimientos médicos se duplican cada 6-8 años, con nuevos procedimientos médicos emergiendo a diario. Mientras que la vida media de los conocimientos médicos es muy corta, un médico practica medicina durante 30 años de media y un enfermero, durante unos 40 años. Por tanto, la formación continuada desempeña un papel clave en la educación de los profesionales de la salud, además de representar un desafío importante a afrontar [1]. Tanto la pirámide de Miller [2] como el cono de aprendizaje de Dale [3] (Figura) plantean una forma muy eficiente de formación del adulto. Miller propone que la curva de aprendizaje de una persona adulta pasa por distintos niveles, desde la adquisición de conocimientos teóricos hasta saber cómo integrar esos conocimientos en la práctica y demostrarlo. Por otro lado, Dale comenta que para recordar un alto porcentaje de esos conocimientos adquiridos hay que realizar una representación teatral o bien simular experiencias reales. La educación a través de la simulación es, por tanto, una respuesta clara a la necesidad de proteger la seguridad del

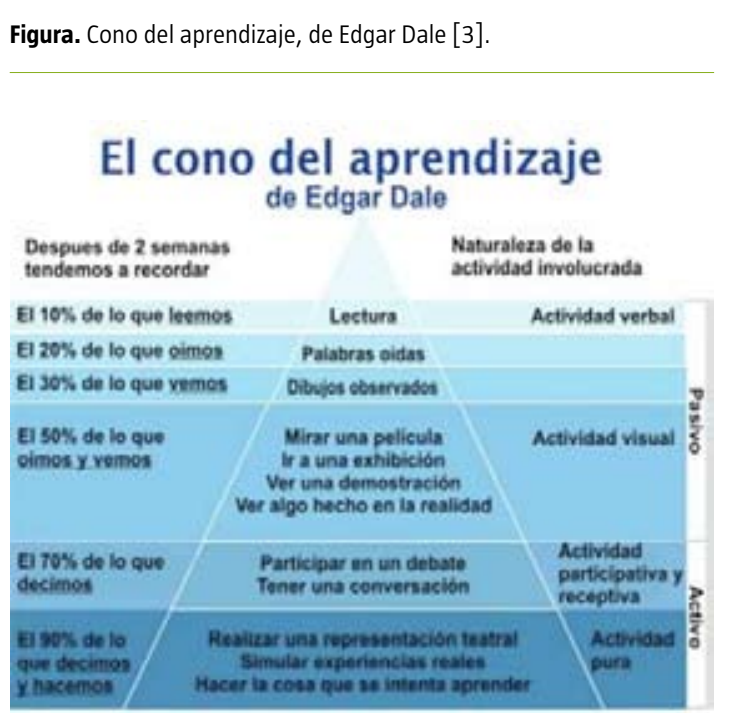

C 2011 Educación Médica

paciente y de garantizar una formación continuada eficiente.

Hoy día, la educación por simulación está muy integrada en la formación de los profesionales de la salud en países como Estados Unidos, Canadá, Reino Unido y Alemania. Esta práctica se realiza mediante el uso de unos equipamientos muy avanzados tecnológicamente y con unas metodologías educativas que han probado ser muy eficientes con la enseñanza por simulación.

Lo más destacado de la enseñanza por simulación es el debriefing [4]. Esta metodología se considera el corazón y el alma de la enseñanza por simulación. Consiste en la revisión autocrítica de todas las actuaciones realizadas durante una experiencia clínica, dirigida por un facultativo.

Diversos estudios [5] han demostrado que la utilización de una metodología de enseñanza efectiva como el debriefing, junto con un equipo avanzado 
tecnológicamente y un profesorado preparado para realizar simulación de forma efectiva [6], mejora sustancialmente la preparación de los estudiantes tanto de pregrado como de posgrado. Con la utilización de la simulación durante el curso académico los profesionales de la salud se enfrentan a la realidad laboral con más confianza y mayor preparación. La simulación también se utiliza en posgrado para reforzar los conocimientos adquiridos con anterioridad, actualizar conocimientos y preparar al profesional para enfrentarse a situaciones poco frecuentes con mayor seguridad.

\section{Bibliografía}

1. Mantovani F, Castelnuovo G, Gaggioli A, Riva G. Virtual reality training for health-care professionals. Cyberpsychol Behav 2003; 6: 389-95.

2. Miller GE. The assessment of clinical skills/competence/ performance. Acad Med 1990; 65 (Suppl 9): S63-7.

3. Dale E. The cone of experience. In Dale E, ed. Audio-visual methods in teaching. New York: Dryden Press; 1946. p. 37-51.

4. Simon R, Rudolph JW, Raemer DB. Debriefing assessment for simulation in healthcare -rater version. Cambridge, MA: Center for Medical Simulation; 2009.

5. Wayne DB, Didwania A, Feinglass J, Fudala MJ, Barsuk JH, McGaghie WC. Simulation-Based education improves quality of care during cardiac arrest team responses at an academic teaching hospital, American College of Chest Physicians. Chest 2008; 133: 156-61.

6. Issenberg SB. The scope of simulation-based healthcare education. Simul Healthc 2006; 1: 203-8.

\section{Comunicaciones libres}

\section{Metodologías educativas}

1.

Kirozainbide: el e-learning quirúrgico

M.A. Durán, I. Arteche, M.T. Maestre, A. Gaztelu Iturri, E. Zurdo, L. Olabe, M.J. Vicente

Hospital de Galdakao-Usánsolo. Vizcaya.

Antecedentes. El proyecto nace en 2003 en el Hospital de Galdakao-Usánsolo con la idea de renovar los protocolos quirúrgicos. Se detectan carencias en la docencia del personal de nueva incorporación y comienza la elaboración de una nueva herramienta online que aúne la transmisión oral y la evidencia científica. Objetivo. Gestionar el conocimiento enfermero para reducir la variabilidad en la práctica quirúrgica mediante la implantación de las TIC. Objetivos específicos: mejorar los protocolos de intervenciones quirúrgicas existentes, evaluando y unificando criterios de actuación; diseñar una herramienta de docencia y formación continuada, utilizando un formato de navegación dinámico, claro, preciso y accesible; y combinar la evidencia científi- ca con la experiencia y el uso de las TIC estructurando equipos de trabajo. Materiales y métodos. Se ha seguido la metodología PDCA (plan, do, check, $a c t$ ) para elaborar una página interactiva en soporte Wikimedia que abarque todo el proceso quirúrgico. Resultados. Se ha obtenido una potente herramienta de aprendizaje y difusión del conocimiento que ha recibido un alto grado de aceptación tanto en el ámbito nacional como internacional. En calidad, Kirozainbide ha sido reconocida como Mejor Buena Práctica de Innovación Euskalit 2010 y EFQM Highly Recommended Innovation Good Practice 2010. Conclusiones. Es una herramienta de gestión del conocimiento que contribuye a la formación continuada del personal, adaptándose a los últimos avances tecnológicos. Útil para la docencia de alumnos de enfermería, en formación de posgrado y personal de nueva incorporación. Es una fuente de conocimiento corporativo, con accesibilidad online desde todos los PC de la red de Osakidetza. Es versátil y aplicable a otros procesos y profesionales.

\section{2.}

Conocimiento generado y adquirido por estudiantes de medicina tras su estancia en salas de espera clínica

D. Solano

Departamento de Medicina. Universidad del País Vasco. Servicio de Medicina Interna. Hospital Universitario de Basurto. Bilbao.

Objetivo. El conocimiento debe situarse en un contexto vivo; es importante dotar de recursos a los alumnos para que por sí mismos generen conocimiento tras observar el medio donde desarrollan su labor. Materiales y métodos. Se muestra la observación reflexiva desarrollada por estudiantes de medicina tras permanecer una hora en salas de espera clínica del hospital donde realizan su formación. La apuesta por dar la palabra al alumno constituye un pilar básico de todo proceso educativo. Resultados. Los alumnos exponen: 'Viendo el comportamiento de los pacientes, te das cuenta de que son personas necesitadas de ayuda, no meras líneas en la hoja de citación diaria.' 'Este ejercicio me ha aportado empatía hacia el enfermo, al conocer y sentir lo que se respira en las salas de espera.' 'He podido observar lo inocente que es la gente cuando espera; no saben lo que les dirá el médico; no lo saben y esperan hasta que el médico les da el resultado o les dice cómo van; no deberíamos olvidarlo cuando nos pongamos definitivamente la bata.' 'Esta vez tenía una misión: observar; espero mantener esta misión siempre, porque observando se apren- 
de y se aprende observando.' 'Me entretiene, me enseña y me ayuda a comprender mejor los sentimientos, sabedor de que nuestro más mínimo gesto puede cambiar la vida de otros para siempre. Conclusiones. Las observaciones seleccionadas aportan una mejor comprensión de la enfermedad, la relación clínica o las instituciones en las que trabajamos. A partir de ellas hemos ido más allá, ofreciendo conocimiento y desarrollando las correspondientes habilidades clínicas.

3.

Diseño e implementación de metodologías activas en una asignatura del área de farmacología en la licenciatura de Medicina

J. Pineda, A. Mendiguren

Departamento de Farmacología. Facultad de Medicina y Odontología. Universidad del País Vasco. Leioa, Vizcaya.

Antecedentes. La llegada del plan de Bolonia a la universidad ha destapado un interés por las metodologías activas y el aprendizaje de competencias académicas y profesionales. Objetivo. El objeto del estudio fue el diseño, implementación y evaluación de la metodología ABP (aprendizaje basado en problemas) en la asignatura 'Farmacología clínica' de quinto curso de la licenciatura de Medicina. La experiencia pretendía desarrollar una competencia específica de la titulación, la prescripción racional de medicamentos esenciales según criterios científicos y éticos sistematizados. Materiales y métodos. En el curso 2009-2010 se programó una actividad grupal APB dentro de los créditos prácticos de la asignatura. La experiencia docente fue analizada mediante los resultados académicos y dos tipos de encuestas de opinión del alumnado (global y específica). Resultados. La actividad práctica se secuenció en cinco sesiones ( $13 \mathrm{~h}$ ), en las que los alumnos se enfrentaban a 12 escenarios clínico-terapéuticos estandarizados y seleccionaban racionalmente la medicación. El proceso tenía tres etapas, nueve indicadores de aprendizaje y finalizaba con la elaboración de un vademécum de medicamentos de primera línea. El sistema de evaluación permitió calificar, de forma continuada y con una prueba final, la adquisición de los indicadores de aprendizaje (50\% de la nota). El 99\% de los alumnos matriculados completaron el proceso ABP, con una alta tasa de éxito y excelencia académica $(n=54)$. Las encuestas de opinión arrojaron promedios superiores a 4 (sobre 5) en todos los ítems analizados $(n=50)$. Como limitación se identificó el número insuficiente de sesiones y horas presenciales. Conclusiones.
El diseño e implementación de una actividad ABP en una asignatura de farmacología ha sido satisfactoria en cuanto a la adquisición de una competencia nuclear de la titulación y la opinión del alumnado. Identificamos como áreas de mejora la gestión del tiempo y la integración.

\section{4.}

Elaboración de casos prácticos para el aprendizaje de la fisiología humana en la titulación de Farmacia

J. Zarate ${ }^{\text {a }}$, M. Gallego a, J. Seco ${ }^{\text {b }}$, B. Calvo ${ }^{\text {a, I. Bar- }}$

bero $^{\text {a }, ~ E . ~ E c h e v a r r i ́ a ~}{ }^{a}$, L.C. Abecia ${ }^{a}$

${ }^{a}$ Universidad del País Vasco. Facultad de Farmacia. Campus de Álava. b Universidad de León. Campus de Ponferrada.

Antecedentes. El nivel de adquisición por parte de los alumnos de las competencias de la asignatura de 'Fisiología' en la titulación de Farmacia depende en gran medida de la metodología de aprendizaje utilizada y de los materiales docentes elaborados por el profesor. Objetivos. Se pretende la creación de un archivo de casos prácticos, que incluyen tanto casos clínicos como situaciones de la vida diaria que requieren adaptaciones de las funciones fisiológicas del organismo, con el fin de que se utilicen en el aula, en la modalidad docente de prácticas de aula. Materiales y métodos. Se ha desarrollado un caso práctico modelo, consistente en la simulación de unas preguntas que podría hacer a su farmacéutico un paciente transexual y su familia. Resultados. El caso comienza así: 'Un niño de 12 años que acude con su madre a tu farmacia refiere que no tiene un sentimiento subjetivo claro de cuál es su sexo; es decir, de si es en realidad un niño o una niña. Esta sensación es constante, lo angustia y le está empezando a plantear algunos problemas.... Conclusiones. Este caso, y otros semejantes, resultan esenciales para que los alumnos se vean en la necesidad de aplicar conceptos fisiológicos, con el fin de alcanzar una mejor comprensión de la materia y utilizarla para resolver problemas reales. La resolución de los casos en el aula permite un aprendizaje cooperativo de los alumnos y un intercambio fluido de información con el profesor, de gran importancia para una titulación sanitaria.

\section{5.}

Análisis de casos clínicos: un enfoque híbrido de transición hacia el aprendizaje activo en la educación en odontología

G. Ibarretxe a , M. Arizmendiarrieta ${ }^{b}$, M. Basaras ${ }^{c}$, I. Pérez-Urcelay ${ }^{d}$,J.J. Azkue ${ }^{e}$ 
a Departamento de Biología Celular e Histología. b Departamento de Estomatología. ${ }^{\mathrm{c}}$ Departamento de Inmunología, Microbiología y Parasitología. d Departamento de Fisiología. e Departamento de Neurociencias. UPV/EHU.

Antecedentes. El aprendizaje activo y basado en competencias es una piedra angular de las reformas curriculares promovidas a través del proceso de Bolonia. Sin embargo, el cambio de sistema de enseñanza desde modelos tradicionales hacia nuevos modelos de educación activa, centrada en los estudiantes, es un reto para cualquier programa educativo. Materiales y métodos. Hemos dado pasos hacia la implementación de metodologías activas en el contexto de un programa convencional de formación en primer curso de Odontología. Durante el segundo período académico del curso 2009-2010 logramos introducir actividades de análisis de casos clínicos (ACC) en paralelo con el programa de enseñanza tradicional en vigor del momento. Notablemente, las actividades de ACC se realizaron con un enfoque multidisciplinar y de manera coordinada entre todas las asignaturas, impartiendo docencia en este periodo. Resultados. La actividad de ACC estimuló el debate entre profesores y compañeros de clase dentro y fuera del aula, promovió el razonamiento crítico en los estudiantes y el aprendizaje autónomo, y estimuló su compromiso y motivación con sus estudios. El grado de satisfacción y la percepción subjetiva de aprendizaje por parte de los estudiantes, evaluados en encuesta anónima al final de curso, fueron muy positivos. Conclusiones. La actividad de ACC es eficaz en combinación con un régimen tradicional de enseñanza para mejorar la adquisición de habilidades de aprendizaje activo y puede considerarse como una forma de transición hacia la plena aplicación de metodologías activas.

6.

Las narraciones digitales como estrategia de innovación educativa

S. Basurto, B. Ruiz de Alegría, E. de Lorenzo

Escuela Universitaria de Enfermería. Vitoria/Gasteiz, Álava.

Antecedentes. Las narraciones digitales son combinaciones del arte de narrar historias añadiendo voz, texto, video y música para presentar o exponer información sobre un tema determinado. Algunos grupos docentes, entre los que cabe destacar el de Bernard Robin, de la Universidad de Houston, han comenzado a utilizar las narraciones digitales aso- ciadas a nuevas metodologías de aprendizaje. Desde el vicerrectorado de innovación docente, en enero del 2009 se pone en marcha el proyecto de innovación 'Utilización de narraciones digitales como recurso de aprendizaje', que pretende explorar las posibilidades de esta herramienta desde un punto de vista pluridisciplinar. Tres profesoras de la escuela de Enfermería de Vitoria se incorporan a este proyecto. Objetivos. Presentar y evaluar la aplicación de las narraciones digitales como estrategia de aprendizaje en la Escuela de Enfermería VitoriaGasteiz, en el curso 2010. Materiales y métodos. Elaboraron una narrativa y un instrumento de evaluación, procediéndose a su implantación y evaluación. Resultados. Se identifica un rango del 70-92\% de los estudiantes que perciben un alto o muy alto grado en el impacto sobre su aprendizaje. El $80 \%$ manifiesta un alto grado de satisfacción con el proceso y un $92 \%$ reconoce que se implica más en la realización de los trabajos. Conclusiones. Tanto docentes como discentes reconocen que la narrativa digital es una estrategia de aprendizaje que potencia la integración del conocimiento a través de mostrar en imágenes conceptos difícilmente transmisibles por la mera palabra. Los estudiantes identifican una mayor implicación en el trabajo y satisfacción con el proceso de aprendizaje.

\section{7.}

Simulación clínica en formación posgraduada en anestesiología y cuidados críticos. Percepción de los médicos residentes

M. Cano, G. Tamayo, M. Pardo, A. Martínez

Servicio de Anestesiología y Reanimación. Hospital de Cruces. Barakaldo, Vizcaya.

Antecedentes. La simulación clínica ha sido reconocida como una estrategia de educación médica que permite el aprendizaje basado en ejercicios próximos a situaciones reales. Objetivos. Evaluar la simulación clínica como herramienta de aprendizaje y adquisición de competencias en el manejo de situaciones críticas. Materiales y métodos. Se evaluó a 20 residentes de Anestesiología y Reanimación de segundo y tercer año por medio de una sesión trimestral durante un periodo de dos años. En cada sesión los residentes se enfrentaban a una situación crítica simulada por medio del simulador a escala real SimMan, que debían resolver durante un tiempo limitado de 20 minutos. Los casos se grababan y posteriormente se discutían. Posteriormente, cada residente debía responder a un cuestionario que evaluaba las siguientes competencias por medio de unos ítems antes y después de los ejercicios: compe- 
tencias relacionadas con habilidades técnicas y conocimientos, competencias de comunicación y trabajo en equipo, liderazgo, gestión de estrés y autoconfianza. Resultados. En cuanto a los ítems que evaluaban las competencias relativas a conocimientos y habilidades, 17 de los 20 residentes afirmaron haber mejorado sus conocimientos. Todos los residentes afirmaron encontrase más seguros en la toma de decisiones y haber mejorado habilidades como la comunicación y la capacidad de trabajo en equipo. Diecisiete afirmaron una mejoría en su capacidad para gestionar el estrés y organizar el trabajo, y 16 habían mejorado su capacidad de liderazgo. Conclusiones. La simulación clínica se perfila como una herramienta de aprendizaje útil no sólo para la adquisición de habilidades técnicas y conocimientos, sino para el desarrollo de competencias tales como la capacidad de liderazgo, la toma de decisiones o las habilidades de comunicación.

\section{8.}

Estilos de aprendizaje de los estudiantes de primer curso de los estudios de biomedicina (CHAEA)

E.G. Argandoña, S. Bulnes, H. Bengoetxea, A. García-Blanco, F.J. Escanero, J.V. Lafuente

Facultad de Medicina. Departamento de Neurociencias. UPV/EHU. Leioa, Bizkaia.

Introducción. El proceso de Bolonia conlleva numerosos cambios en la educación superior en $\mathrm{Eu}$ ropa. Todo cambio debería basarse en un profundo conocimiento de los estudiantes y de sus métodos de estudio. Este trabajo intenta identificar el estilo de aprendizaje predominante en los estudiantes de áreas biomédicas de la UPV-EHU. Materiales y métodos. El estudio se ha llevado a cabo en una muestra de 293 estudiantes de la Escuela de Enfermería y de la Facultad de Medicina durante el curso 2008-2009. El instrumento utilizado ha sido el cuestionario de estilos de aprendizaje (CHAEA) de Honey-Alonso. El análisis de los datos se basó en el contraste de las diferencias de los cuatro estilos a través del test ANOVA del programa SPSS v. 16.0. Resultados. Hay una distribución homogénea de la muestra entre los cuatro estilos explorados. El estilo predominante es el reflexivo, con un valor de 12,7 , siendo ligeramente más alto en los alumnos de medicina (12,9 frente a 12,5). El estilo teórico muestra valores ligeramente más altos en los estudiantes de enfermería (12 frente a 11), siendo el valor medio de 11,5. El estilo pragmático es ligeramente mas bajo que el valor medio $(9,2)$, los valores son similares en ambos grados. El estilo activo es el único que muestra diferencias significativas, siendo 9 para en- fermería y 10 en medicina, con una media de 9,5. Conclusiones. El estilo de aprendizaje predominante en nuestra institución, en esta área, es el reflexivo sobre el activo, siendo significativamente más alto en los estudiantes de enfermería. El resto de los estilos no muestra diferencias significativas.

\section{Posgrado y formación especializada}

\section{9.}

Nuestra experiencia en formación de posgrado: Máster de Oncología Básica y Clínica de la UPV/ EHU

C. de la Hoz, G. López-Vivanco, J.M. Rivera-Pomar Facultad de Medicina y Odontología, UPV/EHU, Leioa. Hospital de Cruces, Osakidetza, Baracaldo, Vizcaya.

Antecedentes. El cáncer comprende un conjunto de enfermedades con características comunes pero con importantes diferencias entre sí. Dada su elevada incidencia y su gravedad, la formación de médicos y otros profesionales en este campo tiene gran interés. Objetivos. El Máster Universitario en Oncología Básica y Clínica de la UPV/EHU, iniciado en 2005, pretende proporcionar a profesionales sanitarios unos conocimientos actuales de los tumores malignos, tanto en el ámbito de la investigación básica como de la actuación clínica. Materiales y métodos. El programa, desarrollado de modo mixto - presencial y online-, consta de 144 temas (www.master-oncologia.es). El material docente se coloca en la plataforma de e-learning de la UPV/ EHU eKASI. Al finalizar el programa, los alumnos realizan un proyecto de fin de máster y un examen test de 60 preguntas. Resultados. Hemos completado dos ediciones de este programa de posgrado con un número de alumnos entre 15 y 20 en cada edición. La mayor parte de los alumnos han sido licenciados en Medicina y residentes de Oncología, pero también hemos tenido otros especialistas, como médicos de atención primaria. El grado de satisfacción de los alumnos es elevado. Conclusiones. Consideramos que esta iniciativa, pionera en la formación de posgrado en ciencias de la salud, contribuye a la formación en oncología, uno de los grandes retos de la medicina actual.

10.

Gestión de tutoría en una unidad de medicina intensiva

J.L. Moreno, I. Seijas, K. Esnaola, F. Labaien

Unidad de Medicina Intensiva. Hospital de Cruces.

Baracaldo, Vizcaya. 
Antecedentes. Debilidad de la tutoría como elemento clave en la formación de especialistas. Objetivo. Impulsar la tutoría como herramienta de calidad en un servicio de medicina intensiva. Adaptación para un programa basado en competencias. (CoBaTrICE). Formar profesionales de garantía y calidad técnica y humana. Materiales y métodos. Nuevo plan de trabajo con los residentes del servicio, potenciación de la tutoría y nombramiento de dos nuevos tutores con diferentes responsabilidades. Ámbito: Servicio de Medicina Intensiva del Hospital de Cruces, Vizcaya. Resultados. Se delimitan nuevas líneas de trabajo a través de dos tutores con funciones diferenciadas, se acotan programas de competencias tanto para residentes propios de la especialidad como para rotantes externos, así como control exhaustivo del programa de aprendizaje y rotaciones. Tutor para residentes de medicina intensiva: diseño de objetivos y competencias (CoBaTrICE), adaptación del programa nacional de la especialidad a nuestro hospital, nuevas formas de trabajo con el residente (libro de recepción, reuniones bimensuales individuales con el tutor, dossier interno, fomentar asistencia a cursos, congresos, etc., así como participación activa en ellos, proyectos de investigación, tesis doctorales). Tutor para residentes rotantes en el servicio: control de rotaciones y objetivos de cada especialidad, control e impulso de sesiones en el servicio. Conclusiones. Tras 14 meses, hemos mejorado de forma significativa la calidad de la formación, así como la confianza que los residentes sienten hacia el servicio y sus componentes.

11.

Factores que influyen en la satisfacción de la formación adquirida por los residentes (MIR) en los servicios del Hospital de Cruces

S. González-Larragán a, J. Morán-Barrios a, L. Martínez ${ }^{\text {b }, ~ A . ~ U r k a r e g i ~ c, ~ M . J . ~ G o n z a ́ l e z-G a r c i ́ a ~ a ~}$

${ }^{a}$ Unidad de Docencia Médica. ${ }^{b}$ Unidad de Investigación. Hospital de Cruces. Baracaldo, Vizcaya. ${ }^{c}$ Departamento de Matemática Aplicada, Estadística e Investigación Operativa. Universidad del País Vasco (EHU/UPV).

Objetivo. Conocer cuáles son los aspectos que más influyen en la formación adquirida por los MIR de los servicios hospitalarios en el año 2010. Comparación con el periodo 2003-2008. Materiales y métodos. Encuesta de 23 preguntas a los MIR del Hospital de Cruces. Niveles de respuesta: muy deficiente (0), deficiente (1), adecuado/bien (2) y excelente (3). Criterio de respuesta: estancia $\geq 2$ meses por el servicio evaluado. Análisis de resultados me- diante la técnica de análisis de correspondencias múltiples (análisis factorial para variables categóricas). Resultados. Tasa de respuestas 2010: 49\% (233/475). Variables que más contribuyen al nivel de percepción de satisfacción: preocupación del servicio por la formación, valoración de la formación docente y supervisión de la labor asistencial. Tiene menor incidencia la valoración de la formación investigadora. Asociación entre realización de sesiones y nivel de satisfacción (más sesiones, mayor satisfacción). Resultados más positivos en residentes cuya rotación ha sido superior a tres meses. Valoraciones más bajas: MIR de Medicina Familiar y Comunitaria (MFyC) comparados con MIR hospitalarios para el mismo tiempo de estancia (dos meses). El análisis de correspondencias múltiples muestra una evolución muy positiva en relación al año 2003. Conclusiones. Clara mejoría en las variables analizadas en 2010 respecto a 2003-2008. Satisfacción de residentes dependiente de la 'preocupación del servicio por su formación, formación docente y supervisión asistencial. La menor satisfacción de los MIR con rotaciones inferiores a tres meses y de MFyC puede relacionarse con la integración, el sentido de pertenencia, la acogida, la aplicación de objetivos del programa y la organización de las tareas formativas.

\section{Evaluación}

\section{2.}

Evaluación de la profesionalidad mediante valoración de $360^{\circ}$. Estudio sobre su viabilidad y aceptación

M. Olmos, B. Forés

Servicio de Anestesiología-Reanimación. Hospital de Basurto. Bilbao.

Antecedentes. La evaluación de la profesionalidad de residentes mediante el método de $360^{\circ}$ implica una comprensión y aceptación del método. Objetivos. Investigar la valoración por parte de los participantes de $360^{\circ}$ de aspectos prácticos, aceptación, justicia y validez del método. Materiales y métodos. Fueron valorados cinco residentes de Anestesiología del Hospital de Basurto que finalizaban su segundo año de formación. Los evaluadores (adjuntos, tutores y otros residentes) completaron un cuestionario de 25 preguntas sobre actitudes y comportamientos, tras lo cual contestaron 10 preguntas dirigidas a valorar aspectos relacionados con su aplicación práctica, aceptación, valor y justicia, con tres niveles de respuesta: positiva, dudosa, negativa. Los residentes evaluados rellenaron el mismo 
cuestionario para autoevaluarse y expresar sus opiniones. Los datos se tabularon mediante el programa SPSS para su análisis estadístico. Resultados. Se recibieron un total de 45 evaluaciones. Cada residente fue evaluado por ocho personas diferentes (dos tutores, dos residentes y cuatro adjuntos) y por él mismo. Conclusiones. Destaca el desconocimiento que los evaluadores tienen sobre los evaluados y la incomodidad relativa con la prueba. Los residentes creen que las respuestas negativas pueden repercutir en las relaciones con el resto de personas. Se cuestiona la honestidad en las respuestas, su utilidad para valorar actitudes y comportamientos y para detectar problemas y solucionarlos. Sólo el 11\% de los evaluadores piensan que la valoración será justa, y ningún residente lo cree totalmente. A la vista de los resultados, pensamos que el $360^{\circ}$ no sería aceptable en nuestro medio. Los motivos pueden ser el desconocimiento de la herramienta, una falta de implicación en el proceso evaluativo o aspectos culturales propios.

\section{Competencias}

13.

Identificación de áreas de mejora en la formación médica por competencias en el periodo de pregrado y en la formación sanitaria especializada

J. Morán-Barrios a, R. Téllez c, L. Martínez b, M.J. González-García ${ }^{\text {a }}$

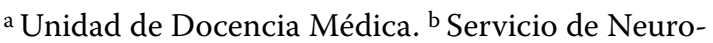
fisiología Clínica. ${ }^{\mathrm{c}}$ Unidad de Investigación. Hospital de Cruces. Baracaldo, Vizcaya.

Objetivos. Conocer la percepción de las competencias (agrupadas en siete dominios), adquiridas, tanto en la formación de pregrado como en la formación sanitaria especializada (FSE). Para ello se han obtenido encuestas de las promociones entrantes en el Hospital de Cruces provenientes de la Universidad del País Vasco entre los años 2004-2009. Asimismo, se han recogido encuestas de la promoción que en 2009 terminó su proceso de FSE. Materiales y métodos. Cuestionario con 34 ítems que se aplicó a 243 médicos en el momento de iniciar el primer año de su programa de FSE, y a un segundo grupo de 44 médicos en el momento de terminarla. Dominios explorados: valores profesionales, actitudes, comportamientos, ética (VPACE); fundamentos científicos de la medicina (FCM); habilidades clínicas (HCLIN); habilidades de comunicación (HCOM); salud pública, sistemas de salud (SPSS); manejo de información (MINF); y análisis crítico, investigación (ACINV). Diseño de pregunta (ejemplo: 'valo- re la competencia adquirida en: redactar historias clínicas...). Escala de respuesta: 0 (nada), 1 (poco), 2 (adecuado-bastante), 3 (mucha). Se analizan las respuestas en cada uno de los dos grupos estudiados. Resultados. Grupo pregrado, 84\% (243/289): VPACE 2,15 (0,56), FCM 2,05 (0,43), HCLIN 1,61 $(0,51)$, HCOM $1,89(0,60)$, SPSS $1,50(0,59)$, MINF 1,42 (0,63), ACINV 1,53 (0,63). Grupo FSE, 100\% (44/44): VPACE 2,42 (0,46), FCM 2,29 (0,45), HCLIN 2,34 (0,48), HCOM 2,40 (0,48), SPSS 1,83 $(0,59)$, MINF 2,14 $(0,49)$, ACINV 2,13 $(0,57)$. Conclusiones. Déficits competenciales: MINF, SPSS, ACINV y HCLIN, en pregrado, y SPSS, ACINV y MINF, en FSE. Esto exige a nuestra organización el desarrollo de programas complementarios al de su propia especialidad.

14.

Formación especializada basada en competencias en el Hospital de Cruces: The CompetencyBased Cruces Hospital Project 2008

J. Morán-Barrios, J. Somme, A. Basterretxea, E. Bereziartua, M. Iriberri, A. Martínez-Berriochoa, M.J. González-García

Unidad de Docencia Médica y Comisión de Docencia. Hospital de Cruces. Osakidetza. Baracaldo, Bizkaia.

Introducción. La formación médica en España (pregrado y especializada) no se basa en competencias. En los años noventa, instituciones de Estados Unidos, Canadá y Reino Unido adoptan un marco basado en competencias para los programas de formación especializada. En 2008, la Unidad de Docencia Médica del Hospital de Cruces introduce un marco de referencia formativo por competencias organizado en siete dominios: profesionalidad, cuidados del paciente, conocimientos, comunicación, promoción de la salud y gestión de recursos, manejo de la información, y análisis crítico e investigación. Objetivos. Introducción de la formación especializada basada en competencias (FEBC) como respuesta a los cambios sociales del siglo XXI. Materiales y métodos. Año 2008: aprobación y exposición del modelo (Comisión de Docencia, Dirección, tutores y jefes de servicio). 2008-2010: seminarios formativos para tutores y residentes, Premio Nacional Cátedra Educación Médica Fundación Lilly 2009, diseño de un portafolio reflexivo por competencias, creación del grupo de expertos en evaluación, inclusión en el plan estratégico del Hospital de Cruces 2010-2014. Resultados. Nuevos métodos de evaluación formativa en siete dominios: formulario para rotaciones y para entrevistas tutorresidente; portafolio reflexivo: de 286 residentes eva- 
luados en 2010, 85\% redactados por competencias y $15 \%$ reflexiones libres. Conclusiones. Definición de la visión docente (ser médico/profesional en el Hospital de Cruces 2008), implementación mediante una estrategia múltiple (plan estratégico, formularios de evaluación y supervisión, y portafolio reflexivo en siete dominios). Es posible poner en práctica la FEBC en residentes sin experiencia previa en sus universidades. Primera experiencia en hospitales españoles de FEBC, según nuestro conocimiento.

15.

Adquisición de competencias del área de la radiología y medicina física en medicina mediante metodologías activas

T. Palomares, J. González, P. Bilbao

Departamento de Cirugía y Radiología y Medicina Física. Facultad de Medicina y Odontología. UPV/EHU. Leioa, Bizkaia.

Antecedentes. Una de las competencias fundamentales en la formación de un médico es el adecuado análisis e interpretación de imágenes radiológicas. Objetivos. Desarrollar las competencias específicas de la radiología en medicina mediante una metodología activa dirigida al autoaprendizaje reflexivo, cooperativo en línea con la filosofía IKD de la UPV/EHU. Materiales y métodos. Los alumnos de tercer curso de la licenciatura en Medicina, dentro de la asignatura de 'Radiología y medicina física generales', establecidos en grupos de trabajo, realizaron el análisis de problemas y la búsqueda de información significativa para conseguir habilidades médicas que les permitieran resolverlos de forma cooperativa. A través de la plataforma informática e-kasi se dispusieron recursos digitales (soporte de documentos PDF con el argumento del problema, imágenes radiológicas e interrogantes a resolver). Los grupos de trabajo entregaron un informe para su evaluación. Se realizó una encuesta a los alumnos para conocer su opinión del proceso. Resultados. Los alumnos realizaron una búsqueda optimizada de la información médica para la adquisición de conocimientos necesarios para la resolución de problemas, redactaron informes con utilización de la terminología apropiada, una correcta sistemática de lectura e interpretación metodológica de las técnicas de imagen y la correlación de síntomas clínicos con los radiológicos. Sobre la innovación docente desarrollada, los alumnos opinan que ha favorecido el aprendizaje de la asignatura y el desarrollo de competencias generales. Conclusiones. El aprendizaje cooperativo permite adquirir de un modo eficaz competencias del área de la radiología general.
16.

Evaluando las competencias médicas en psiquiatría M.A. Ortiz-Jauregi

Departamento de Neurociencias-Psiquiatría. Facultad de Medicina y Odontología. UPV/EHU. Leioa, Vizcaya.

Los estudios de medicina, tanto de pregrado, posgrado como de educación continuada, implican un proceso continuo de adquisición, mantenimiento y mejora de habilidades para la práctica de la profesión. La adaptación al Espacio Europeo de Educación Superior en la enseñanza-aprendizaje de los procedimientos médicos genera un interés añadido en la búsqueda de nuevos métodos de formación en habilidades médicas y su evaluación. Este enfoque a la enseñanza-aprendizaje de las competencias sanitarias lleva a un cambio de paradigma en la propia enseñanzaaprendizaje y en los métodos de evaluación de la formación y las competencias profesionales de la medicina. Entre las estrategias de enseñanza-aprendizaje más relevantes de competencias en psiquiatría encontramos el aprendizaje basado en problemas, el aprendizaje basado en casos, los informes temáticos, la investigación bibliográfica, la elaboración de mapas conceptuales y mentales, las lecturas facilitadas de textos y los seminarios temáticos. En este marco, vamos a tratar de mostrar el resultado de un método antiguo renovado de evaluación de la adquisición de conocimientos psiquiátricos en la formación médica basada en la evaluación clínica objetiva y estructurada (ECOE), como método de evaluación de habilidades médicas clínicas relacionadas con las prácticas más procedimentales, de comunicación y habilidades, y como método que combina distintos recursos metodológicos de evaluación en un intento de alcanzar una evaluación estandarizada y objetiva.

17.

La estética en el cuidado. Una ética y una competencia profesional

J. Lobo Delgado

Unidad Neonatal. Hospital de Cruces. Barakaldo, Bizcaia.

Antecedentes. La aparición de trabajos profesionales que intentan superar el reduccionismo de los enfoques positivistas, cognitivistas, y la aparición de aproximaciones fenomenológicas existenciales, prácticas reflexivas y trabajos etnometodológicos, nos invitan a construir una ciencia humana del cuidado. Objetivo. Comprender las condiciones de emergencia y características del discurso estético, entendiendo por discurso no sólo los significados transmitidos por el lenguaje, sino las prácticas que contribuyen a 
producir los significados subjetivos de los profesionales. Materiales y métodos. La base teórica que da soporte a esta indagación la constituye la fenomenología herméutica de Gadadmer, donde el lenguaje se convierte en central, además de ser el medio de la experiencia herméutica y la filosofía del arte de Dewey, que insiste en la comprensión del rol de las emociones y los sentimientos en la creación de una experiencia. Es desde aquí donde la fenomenología de van Manen se ofrece como el camino más coherente al objetivo señalado. Resultados. En la relación paciente-profesional se dan y reciben contenidos que son de una naturaleza distinta al conocimiento cognitivo-técnico. Es un conocimiento de naturaleza accional, situacional y corporal, que reside en nuestras acciones e implica un conocimiento experto de la ciencia y el arte. Estos aspectos creativos y estéticos sólo pueden ser traducidos por el profesional a partir de sus experiencias, que le permiten diseñar estrategias para su intervención. En este proceso se pone de manifiesto el valor del arte de cuidar. Conclusión. Consideramos necesario que los procesos de formación de los profesionales de ciencias de la salud, tanto de grado como de posgrado, contemplen entre sus competencias aquellas que permitan desarrollar y potenciar la estética en el cuidado. Esto contribuiría a construir un nuevo conocimiento generador de respuestas que sirvan de vía de transformación de conocimiento y de construcción de organizaciones sanitarias más humanizadas.

18.

Seguridad del paciente. Declaración voluntaria de incidentes

J. López de Heredia, M.C. López-Herrera, A. Campino, A. Valls i Soler

Unidad Neonatal. Hospital de Cruces. Barakaldo, Bizcaia.

Antecedentes. La seguridad del paciente por los errores médicos ha pasado de ser una cuestión ignorada y ocultada a convertirse en un elemento clave en la política sanitaria. Así, las publicaciones científicas y la inversión en seguridad del paciente han crecido de forma exponencial, reflejando la preocupación e importancia de este tema. El objetivo prioritario es reducir el número de errores, su impacto sobre la salud de los pacientes y los costes de la atención sanitaria. Uno de los puntos clave para mejorar la seguridad del paciente es conocer los errores asociados a la práctica médica, registrarlos y analizar su génesis para tomar medidas y cambiar conductas que eviten o al menos disminuyan su número y consecuencias negativas. Objetivo. Presentar un sistema de recogida de errores en la Unidad Neonatal del Hospital de
Cruces durante los últimos cuatro años. Resultados. En dicho periodo se declararon de forma anónima y voluntaria 350 'errores médicos'. El 42\% fueron incidentes que no afectaron al paciente y el $50 \%$ se asociaron a medidas terapéuticas. En cuatro casos apareció algún tipo de secuelas y no se produjo ningún fallecimiento. Se analizan las prácticas inseguras y los factores asociados al evento que favorecen su presentación. Conclusiones. Los sistemas de recogida y análisis de incidentes son útiles para mejorar la seguridad de los pacientes, debiendo introducirse alguno de sus aspectos en los programas de formación.

\section{Pregrado}

\section{9.}

Canales preferenciales utilizados por los alumnos de primer curso de carreras biomédicas para la adquisición de información (VAK)

J.V. Lafuente, H. Bengoetxea, S. Bulnes, A. GarcíaBlanco, E.G. Argandoña, G. Díaz-Veliz

Facultad de Medicina. Departamento de Neurociencias. UPV/EHU. Leioa, Bizkaia.

Introducción. Para la adquisición de la información durante el proceso de aprendizaje todos utilizamos un canal con preferencia sobre los demás. El conocimiento de este aspecto puede ser muy importante para facilitar la tarea de enseñanza-aprendizaje. El objetivo de este estudio ha sido identificar el canal utilizado preferentemente por los alumnos de dos estudios biomédicos en la Universidad del País Vasco/EHU. Materiales y métodos. El estudio se llevó a cabo en una muestra de 143 alumnos pertenecientes a la Facultad de Medicina y a la Escuela de Enfermería durante el curso 2008-2009. El instrumento usado fue el test 'Visual, Auditivo y Cinéstesico' (VAK), adaptado por Romo y López. El análisis de los datos se basó en el análisis de las diferencias entre las medias para los tres canales analizados mediante el test de ANOVA (SPSS v. 16.0). Resultados. Se pone de manifiesto una distribución homogénea de la muestra entre los tres canales explorados. El valor medio fue alrededor de 18 puntos para visuales y auditivos y de 16 para cinestésicos, tanto para los alumnos de medicina como para los alumnos de enfermería. La única diferencia significativa entre grados fue para el canal visual, significativamente preferido por estudiantes de enfermería. Conclusiones. Al comienzo de los estudio biomédicos, los alumnos muestran escasas diferencias respecto a los canales utilizados preferentemente para la adquisición de la información. Los estudiantes de enfermería utilizan preferentemente el canal visual. 